\title{
5-Methoxysalicylic Acid Matrix for Ganglioside Analysis with Matrix-Assisted Laser Desorption/Ionization Mass Spectrometry
}

\author{
Dongkun Lee, Sangwon Cha
}

Department of Chemistry, Hankuk University of Foreign Studies, Yongin, 449-791, Korea

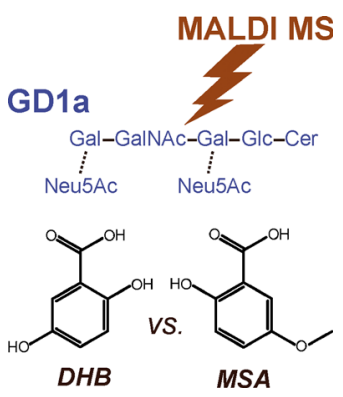

Abstract. In this note, we report that high quality ganglioside profiles with
minimal loss of sialic acid residues can be obtained in the positive ion mode
by using a 5-methoxysalicylic acid (MSA) matrix for matrix-assisted laser
desorption/ionization (MALDI) mass spectrometry (MS). Our results showed
that MSA produced much less sialic acid losses from gangliosides than DHB,
although MSA and $\mathrm{DHB}$ are differ only by their functional groups at their 5-
positions (-OH for $\mathrm{DHB}$ and $-\mathrm{OCH}_{3}$ for MSA). Furthermore, our data also
demonstrated that addition of an alkali metal additive was effective for sim-
plifying ganglioside profiles, but not necessary for stabilizing glycosidic bonds
of gangliosides if MSA was used as a matrix. This suggests that MALDI MS

with MSA has a potential to gain additional benefits from the positive-ion mode analyses without losing performance in ganglioside profiling.

Keywords: MALDI, 5-Methoxysalicylic acid, Ganglioside, Sialic acid, Alkali metal

Received: 10 July 2014/Revised: 22 October 2014/Accepted: 26 October 2014/Published Online: 11 December 2014

\section{Introduction}

$\mathrm{G}$ angliosides, glycosphingolipids with one or more sialic acid residues, are one of the labile lipids undergoing severe loss of sialyl groups during MALDI process. This often makes it difficult to estimate relative abundances of gangliosides from complex samples and also results in misleading localization information in MALDI MS imaging of gangliosides, as Colsch et al. pointed out [1]. Therefore, many research efforts have been made to stabilize ganglioside ions during MALDI process.

First, high-pressure MALDI ion sources have been successfully developed to cool vibrationally excited labile gangliosides [2-5]. Second, various MALDI matrixes have been tested for finding the best "cold" matrix [3, 6-10]. In several initial reports, 2,5-dihydroxybenzoic acid (DHB) [3, 6, 8] and 6aza-2-thiothymine (ATT) [3, 6] were found to be reasonably "cold" matrixes for ganglioside detection. Recently, other engineered matrixes have been reported to perform better than DHB or ATT. Li et al. found that an ionic liquid matrix, 1-

Electronic supplementary material The online version of this article (doi:10.1007/s13361-014-1037-2) contains supplementary material, which is available to authorized users.

Correspondence to: Sangwon Cha; e-mail: swcha@hufs.ac.kr methylimidazolium $\alpha$-cyano-4-hydroxycinnamate (ImCHCA) [9], produced much less fragmentation for gangliosides than DHB did [9]. Colsch and Woods also developed a novel matrix mixture, 2,6-dihydroxyacetophenone (DHA)/ammonium sulfate/heptafluorobutyric acid (HFBA), for maximizing ganglioside detection from a complex mixture $[1,10]$. Ammonium salt in the mixture effectively reduced spectral complexity as reported previously [11] and HFBA increased vacuum stability of DHA enough to perform MALDI imaging experiments [10]. Third, Lebrilla's group found that addition of large alkali metal ions such as cesium ions could decrease the glycosidic bond cleavages by steric crowding effect and, thus, stabilize gangliosides [12, 13]. Recently, Woods et al. also stabilized gangliosides with cesium ions in MALDI-ion mobility MS analysis of gangliosides in the positive ion mode [14].

In this note, we report that 5-methoxysalicylic acid (MSA) is an effective "cold" matrix for detecting gangliosides, especially in the positive ion mode. Usually, it is much more difficult to detect intact gangliosides in the positive ion mode than in the negative ion mode because cation-induced fragmentation of sialic acid groups is significant [13]. However, this was not the case with MSA. MSA has long been used as a component of super-DHB (sDHB, 9:1 w/w DHB/MSA) [14, $15]$ and DHB/MSA/fucose [16] matrixes. MSA with spermine was also found to be a useful matrix in the MALDI MS analysis 
of oligonucleotides [17]. However, the use of MSA alone for gangliosides has not been reported. MSA produced ganglioside profiles with minimal loss of sialic acids in the positive ion mode, without aid of other stabilizing conditions. In addition, MSA supplemented with a proper alkali metal salt produced simple and sensitive ganglioside signatures.

\section{Experimental}

Porcine brain total ganglioside extract and bovine brain GD1a extract were purchased from Avanti Polar Lipids (Alabaster, AL, USA) and Wako Pure Chemical Industries (Osaka, Japan), respectively. Chloride salts of alkali metals $(\mathrm{NaCl}, \mathrm{KCl}, \mathrm{RbCl}$, and $\mathrm{CsCl}$ ), DHB, sDHB, and MSA were obtained from SigmaAldrich. (St. Louis, MO, USA). All solvents except deionized water were purchased from Fisher Scientific (Fairlawn, NJ, USA).

Stock solutions $(1.0 \mathrm{mg} / \mathrm{mL})$ of total brain ganglioside extract and brain GD1a extract were prepared in chloroform/ methanol $(1: 1, \mathrm{v} / \mathrm{v})$ and diluted to various concentrations for MALDI MS analysis. MALDI matrix solutions $(10 \mathrm{mg} / \mathrm{mL})$ were prepared in methanol/water $(4: 1, \mathrm{v} / \mathrm{v})$. When a salt additive was added to a matrix solution, final concentration of an additive in a matrix solution was $20 \mathrm{mM}$ for an alkali salt and $3 \mathrm{mM}$ for ammonium sulfate. For MALDI MS analysis, the ganglioside extract samples $(0.5 \mu \mathrm{L})$ was first spotted onto a 384-well MALDI target plate (ASTA Inc., Suwon, Korea) followed by $0.5 \mu \mathrm{L}$ MALDI matrix. After spotting, the MALDI target was gently dried under moderate vacuum ( $\sim 50$ Torr) at ambient temperature.

MALDI MS analysis was performed using ABI 4800 Plus MALDI-TOF/TOF analyzer (Applied Biosystems, Foster City, CA, USA) equipped with a $355 \mathrm{~nm} \mathrm{Nd:YAG} \mathrm{laser} \mathrm{and} \mathrm{con-}$ trolled by the 4000 Series Explorer software. The mass spectrometer was operated in the positive or negative reflectron mode with a $20 \mathrm{kV}$ acceleration voltage. For a given sample spot, sub-spectra obtained from 40 different locations in the spot were averaged to a final mass spectrum (800 laser shots/spot). DataExplorer ver. 4.8 (Applied Biosystems) and mMass (http://www.mmass.org) were used for postprocessing mass spectra.

\section{Results and Discussion}

\section{GDla Analysis Using MSA}

In order to evaluate the performance of the various MALDI matrixes in detecting gangliosides, we used a bovine GD1a extract containing two major disialogangliosides illustrated in Supplementary Figure S1. During evaluation, we observed an interesting difference in ganglioside profiles obtained by DHB and SDHB matrixes in the positive ion mode as shown in Figure 1a and $b$. Although intact GD1a ions could be detected with DHB, loss of $N$-acetylneuraminic acid (Neu5Ac) residues was significant, and asialoganglioside ions resulting from
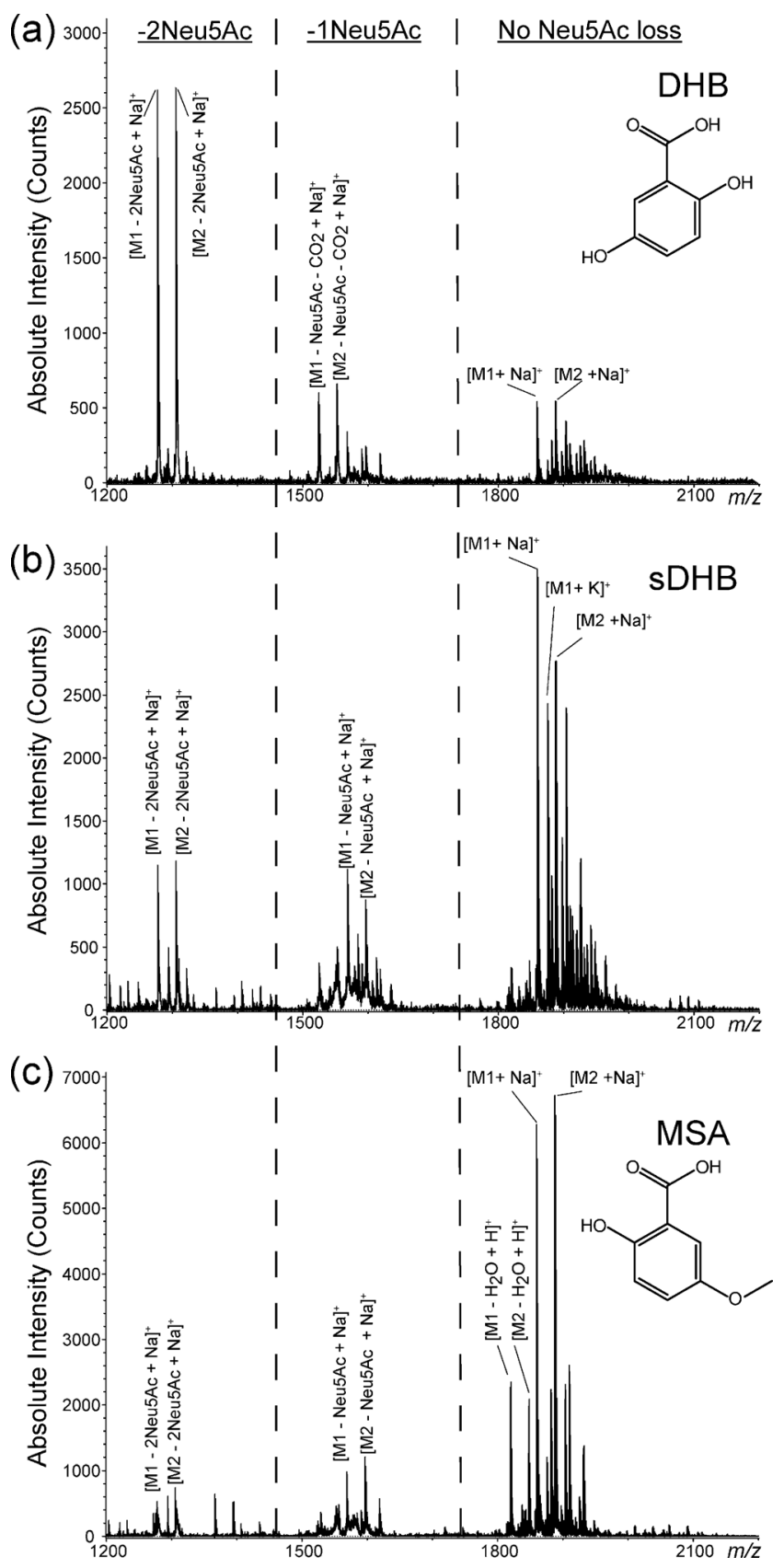

Figure 1. MALDI mass spectra of a bovine brain GD1a extract (0.5 $\mathrm{\mu g}$ per spot) with (a) DHB, (b) $\mathrm{sDHB}$, and (c) MSA as a matrix. M1: GD1a (d18:1/18:0), M2: GD1a (d20:1/18:0). See Supplementary Figure $\mathrm{S} 1$ for the chemical structures and Supplementary Table S1 for detailed peak assignments with relative intensities

losing two Neu5Ac residues were dominant species in the spectrum (Figure 1a). In contrast, sDHB mainly generated intact GD1a ions, and Neu5Ac fragmentation was significantly reduced (Figure 1b). Since sDHB differs from DHB only by the presence of MSA, we assumed that MSA gives rise to the aforementioned difference in ganglioside profiles. Therefore, we tested MSA alone as a MALDI matrix for ganglioside detection. Compared with sDHB, the MSA-only matrix further 
reduced the amount of Neu5Ac loss and enhanced signals from intact GD1a (Figure 1c). These observations indicate that MSA induces much less amount of charge-induced glycosidic bond cleavages than DHB does in the positive ion mode, although the functional groups of DHB and MSA differ only at their 5position ( $-\mathrm{OH}$ for DHB and $-\mathrm{OCH}_{3}$ for MSA). Hence, the positive-ion mode ganglioside profile obtained by MSA could reflect the relative abundances of different gangliosides from a complex mixture more precisely, whereas the DHB profile is near impossible to interpret (Supplementary Figure S2). In the negative ion mode, however, difference in the degree of Neu5Ac loss between MSA and DHB was not as substantial as in the positive ion mode, although major ions detected were remarkably different from each other (Supplementary Figure S3).

There are potential advantages of ganglioside detection in the positive ion mode if the positive ion mode could exhibit comparable sensitivity and softness to the negative ion mode. First, the positive ion mode can detect the asialoganglioside fragment ions, which is undetectable in the negative ion mode. These additional ganglioside signatures can be used as a normalization factor for presenting correct localization of gangliosides in MALDI MS imaging experiments, similar to the previous study [1]. Second, tandem mass spectra of gangliosides in the positive ion mode have shown to provide more informative fragment patterns of glycans and ceramides than those in the negative ion mode [15]. Third, the positive ion mode allows simultaneous detection of neutral lipids and acidic lipids by forming ions through cationization.

\section{Effects of Salt Additives in MALDI MS of Gangliosides with MSA}

Although we found MSA alone as an effective matrix for detecting intact gangliosides (see Figure 1c), several challenges remained in profiling gangliosides with MSA in the positive ion mode. First, unfocused post-source decay (PSD) fragment ions of GD1 a were observed around $\mathrm{m} / \mathrm{z} 1500-1600$, but these PSD fragment ions were easily eliminated by decreasing relative concentration of gangliosides to the MSA. Second, a significant amount of water-loss fragment ions were generated. Third, adduct ions formed by several alkali metals increased spectral complexities. The last two issues could be overcome by adding an appropriate salt additive to the MSA matrix solution as indicated by previous reports on ammonium salts simplifying adduct ion formation of gangliosides [10, 11, 14] and alkali metal salts, especially cesium salts, stabilizing ganglioside ions [12-14]. Supplementary Figure S4 demonstrates the effects of the ammonium salt additive in detecting gangliosides with MSA. With ammonium sulfate, spectral complexity was significantly reduced by removing potassium adduct ions. However, ammonium sulfate could not diminish the formation of water-loss fragment ions.

The effects of various alkali metal salt additives are presented in Supplementary Figure S5 and summarized in Table 1. First, all alkali metal salts effectively suppressed other alkali
Table 1. Relative Ion Intensities (\%) of GD1a (d18:1/18:0) (M1)-Derived Ions in MALDI Mass Spectra of a Bovine Brain GD1a Extract with MSA and Various Alkali Metal Additives. See Supplementary Figure S5 for Mass Spectra

\begin{tabular}{|c|c|c|c|c|}
\hline \multirow[t]{2}{*}{ Ions detected } & \multicolumn{4}{|c|}{ Alkali metal additive } \\
\hline & $\mathrm{NaCl}$ & $\mathrm{KCl}$ & $\mathrm{RbCl}$ & $\mathrm{CsCl}$ \\
\hline$\left[\mathrm{M} 1-2 \mathrm{Neu} 5 \mathrm{Ac}+\right.$ metal $^{+}$ & 0.6 & 0.8 & 3.3 & 1.9 \\
\hline$\left[\mathrm{M} 1-\right.$ Neu $5 \mathrm{Ac}+$ metal $^{+}$ & 8.0 & 5.7 & 9.1 & 9.8 \\
\hline$[\mathrm{M} 1-\mathrm{Neu} 5 \mathrm{Ac}+2 \mathrm{metal}]^{+}$ & 4.5 & 2.2 & 5.5 & 11.6 \\
\hline$\left[\mathrm{M} 1-\mathrm{H}_{2} \mathrm{O}+\text { metal }\right]^{+}$ & 6.0 & 5.4 & 12.1 & 6.7 \\
\hline$[\mathrm{M} 1+\text { metal }]^{+}$ & 100.0 & 100.0 & 100.0 & 100.0 \\
\hline$[\mathrm{M} 1+2 \text { metal }]^{+}$ & 47.5 & 46.8 & 46.1 & 58.3 \\
\hline$[\mathrm{M} 1+3 \text { metal }]^{+}$ & 32.7 & 18.5 & 29.1 & 62.0 \\
\hline
\end{tabular}

metal adduct ions; therefore, spectral complexity was noticeably reduced. Second, MSA plus an alkali chloride salt generated much less or no water-loss fragment ions from GD1a than MSA-only or MSA with ammonium sulfate did. Third, however, we did not observe further significant stabilization of glycosidic bonds by an alkali salt additive. In addition, we also could not find the correlation between glycosidic bond stabilities and metal ion sizes [13]. This may be because the MSA matrix alone is enough to stabilize ganglioside ions up to disialo-species at least. Fourth, consistent with the previous report [16], a sialic acid residue tends to form a complex with large cesium ions more easily than other alkali metal ions. These observations indicate that all alkali metal salts, regardless of their sizes, generally worked well with the MSA matrix for simplifying profiles and for reducing water-loss fragment ions. Nonetheless, we chose potassium salts as the best among those tested based on two observations: (1) the $\mathrm{m} / \mathrm{z}$ spacing produced by potassium adduct ion formation prevented ganglioside profiles from crowding in the narrow $\mathrm{m} / \mathrm{z}$ region; (2) potassium salts produced ganglioside signatures with the highest reproducibility and the mid-femtomole detection limit (500 fmol/spot for $[\mathrm{M}+\mathrm{K}]^{+}$ion with $\mathrm{S} / \mathrm{N}$ ratio of 10$)$. As shown in Supplementary Figure S6, we obtained clear ganglioside profiles up to trisialogangliosides (GT) from a complex total ganglioside extract in the positive ion mode by using MSA with $\mathrm{KCl}$.

\section{Conclusions}

In this note, we report that MSA could be a matrix of choice for profiling gangliosides in the positive ion mode. MSA alone showed its exceptional softness in the ionization of the gangliosides and also worked well with any alkali salt additive for spectral simplification. Although it is hard to explain the reason for the difference between DHB and MSA based on data presented here, we may get possible clues from their physicochemical properties summarized in Supplementary Information Table S2. As expected from their structural similarities, proton affinities, acidities, and UV absorption characteristics of DHB and MSA are very similar to each other. On the other hand, MSA has a significantly lower melting point $(\sim 412$ versus $\sim 475 \mathrm{~K})$ and a higher vapor pressure $\left(2.09 \times 10^{-5}\right.$ versus 
$2.10 \times 10^{-6} \mathrm{mmHg}$ at $298 \mathrm{~K}$ ) than DHB. These physical properties generally reflect the intermolecular interaction forces and also have been correlated to the softness of MALDI [17, 18]. For example, since the matrix melting points can be roughly correlated to the matrix crystal hardness [18], a matrix with a lower melting point may form less hard crystals, require less energy for ablation, generate colder plume, and therefore induce less in-source fragmentation than one with a higher melting point $[18,19]$. However, this is only one of the many possible explanations. Intensive investigations on chemical properties of these matrixes in a condensed phase and characterization of their ablation behaviors are at least required to elucidate this phenomenon.

\section{Acknowledgments}

The authors acknowledge support for this research by Basic Science Research Program through the National Research Foundation of Korea (NRF) funded by the Ministry of Science, ICT, and Future Planning (NRF-2013R1A1A1010947).

\section{References}

1. Colsch, B., Jackson, S.N., Dutta, S., Woods, A.S.: Molecular microscopy of brain gangliosides: Illustrating their distribution in hippocampal cell layers. ACS Chem. Neurosci. 2, 213-222 (2011)

2. O'Connor, P.B., Costello, C.E.: A high pressure matrix-assisted laser desorption/ionization Fourier transform mass spectrometry ion source for thermal stabilization of labile biomolecules. Rapid Commun. Mass Spectrom. 15, 1862-1868 (2001)

3. Ivleva, V.B., Elkin, Y.N., Budnik, B.A., Moyer, S.C., O’Connor, P.B., Costello, C.E.: Coupling thin-layer chromatography with vibrational cooling matrix-assisted laser desorption/ionization Fourier transform mass spectrometry for the analysis of ganglioside mixtures. Anal. Chem. 76, 6484-6491 (2004)

4. O’Connor, P., Budnik, B., Ivleva, V., Kaur, P., Moyer, S., Pittman, J., Costello, C.: A high pressure matrix-assisted laser desorption ion source for Fourier transform mass spectrometry designed to accommodate large targets with diverse surfaces. J. Am. Soc. Mass Spectrom. 15, 128-132 (2004)

5. Zhang, J., LaMotte, L., Dodds, E.D., Lebrilla, C.B.: Atmospheric pressure MALDI Fourier transform mass spectrometry of labile oligosaccharides. Anal. Chem. 77, 4429-4438 (2005)
6. Juhasz, P., Costello, C.: Matrix-assisted laser desorption ionization time-offlight mass spectrometry of underivatized and permethylated gangliosides. J. Am. Soc. Mass Spectrom. 3, 785-796 (1992)

7. Juhasz, P., Costello, C.E., Biemann, K.: Matrix-assisted laser desorption ionization mass spectrometry with 2-(4-hydroxyphenylazo)benzoic acid matrix. J. Am. Soc. Mass Spectrom. 4, 399-409 (1993)

8. Sugiyama, E., Hara, A., Uemura, K.-I., Taketomi, T.: Application of matrix-assisted laser desorption ionization time-of-flight mass spectrometry with delayed ion extraction to ganglioside analyses. Glycobiology 7, 719724 (1997)

9. Chan, K., Lanthier, P., Liu, X., Sandhu, J.K., Stanimirovic, D., Li, J.: MALDI mass spectrometry imaging of gangliosides in mouse brain using ionic liquid matrix. Anal. Chim. Acta. 639, 57-61 (2009)

10. Colsch, B., Woods, A.S.: Localization and imaging of sialylated glycosphingolipids in brain tissue sections by MALDI mass spectrometry. Glycobiology 20, 661-667 (2010)

11. Ueki, M., Yamaguchi, M.: Analysis of acidic carbohydrates as their quaternary ammonium or phosphonium salts by matrix-assisted laser desorption/ionization mass spectrometry. Carbohydr. Res. 340, 17221731 (2005)

12. Cancilla, M.T., Penn, S.G., Carroll, J.A., Lebrilla, C.B.: Coordination of alkali metals to oligosaccharides dictates fragmentation behavior in matrix assisted laser desorption ionization/Fourier transform mass spectrometry. J. Am. Chem. Soc. 118, 6736-6745 (1996)

13. Penn, S., Cancilla, M., Green, M., Lebrilla, C.: Direct comparison of matrix-assisted laser desorption/ionisation and electrospray ionisation in the analysis of gangliosides by Fourier transform mass spectrometry. Eur. J. Mass Spectrom. 3, 67-79 (1997)

14. Jackson, S.N., Colsch, B., Egan, T., Lewis, E.K., Schultz, J.A., Woods, A.S.: Gangliosides' analysis by MALDI-ion mobility MS. Analyst 136, 463-466 (2011)

15. Lee, H., An, H.J., Lerno Jr., L.A., German, J.B., Lebrilla, C.B.: Rapid profiling of bovine and human milk gangliosides by matrixassisted laser desorption/ionization Fourier transform ion cyclotron resonance mass spectrometry. Int. J. Mass Spectrom. 305, 138-150 (2011)

16. Penn, S.G., Cancilla, M.T., Lebrilla, C.B.: Fragmentation behavior of multiple-metal-coordinated acidic oligosaccharides studied by matrix-assisted laser desorption ionization Fourier transform mass spectrometry. Int. J. Mass Spectrom. 195/196, 259-269 (2000)

17. Knochenmuss, R.: Ion formation mechanisms in UV-MALDI. Analyst 131, 966-986 (2006)

18. Bae, Y., Moon, J., Kim, M.: Expansion cooling in the matrix plume is under-recognized in MALDI Mass spectrometry. J. Am. Soc. Mass Spectrom. 22, 1070-1078 (2011)

19. Hillenkamp, F., Karas, M.: The MALDI Process and Method. MALDI MS, pp. 1-28. Wiley-VCH Verlag GmbH: KGaA, Weinheim (2007) 